J3eA, Journal sur l'enseignement des sciences et technologies de l'information et des systèmes, Volume 4, Hors-Série 2, 23 (2005)

DOI : http://dx.doi.org/10.1051/bib-j3ea:2005723

(C) EDP Sciences, 2005

Dessin et simulation de fonctions de base en CMOS $90 \mathrm{~nm}$

E. Sicard et S. Ben Dhia

INSA, Département de Génie Électrique et Informatique

135 avenue de Rangueil

F-31077 Toulouse CEDEX 4, France 


\title{
Dessin et simulation de fonctions de base en CMOS 90nm
}

\author{
E. Sicard, S. Ben Dhia \\ INSA, Département de Génie Electrique et Informartique, 135 av. de Rangueil 31077 \\ Toulouse cedex 4, France. \\ etienne.sicard@insa-tlse.fr \\ http://intrage.insa-tlse.fr/ etienne
}

\section{Résumé :}

Cet article présente le dessin des masques et la simulation analogique de cellules de base en technologie CMOS 90 nanomètres. Le logiciel Microwind, didacticiel de dessin sur PC inclut différents modules pédagogiques permettant de comprendre les enjeux des nouvelles technologiques CMOS nanométriques: visualisation 2D et 3D du procédé d'intégration, propriétés et caractéristiques des transistors, effets parasites des canaux ultra-courts, fonctionnement des cellules logiques de base, principe d'assemblage des fonctions arithmétiques, construction des mémoires ROM, SRAM, DRAM, Flash et FRAM, construction des fonctions analogiques telles que VCO, mixers et convertisseurs. L'utilisation du logiciel didactique est aussi détaillée.

Mots clés : CMOS, 90nm, logique, mémoire, dessin des masques, simulation analogique

\section{INTRODUCTION}

La course effrénée à la performance en microélectronique se traduit par des efforts technologiques colossaux permettant de doubler les performances des circuits intégrés tous les trois ans. L'augmentation parallèle du coût est si forte que les fabricants des puces doivent se regrouper et concentrer leurs centrales technologiques. Tel est le cas de l'association entre Motorola, STMicroelectronics, Philips et TSMC à Crolles. Les limites physiques à la réduction des dimensions sont sans cesse repoussées, comme le suggère la figure 1 . La plupart des analystes s'accordent sur la mise au point de la technologie 9nm vers 2015-2020.

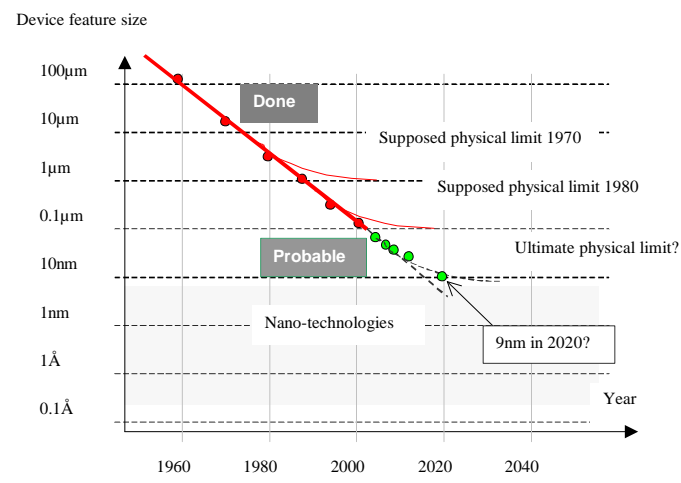

fig 1: Les progrès en intégrations laissent supposer une lithogravure 9nm en 2020 [1]

L'éducation en micro-électronique pose différents problèmes :

- La vie d'un cours de micro-électronique est courte. Les concepts de base sont ébranlés par l'évolution technologique et doivent évoluer.

- La vie d'un exercice pratique est encore plus courte. Les logiciels utilisés évoluent de façon majeure tous les deux ans, de façon mineure tous les six mois.

- L'accès à la connaissance est difficile. Les livres sont souvent en décalage par rapport aux besoins. L'information technologique de pointe reste confidentielle et se monnaye cher.

L'éducation en micro-électronique n'est plus le seul domaine des écoles d'ingénieurs et des Universités. Les entreprises doivent faire face au recyclage continu de leur employés, aux évolutions technologiques, menant certains fabricants jusqu'à créer leur propres universités dont le succès est vital pour la compétitivité, voire la survie de l'entreprise. Les technologies liées au multimédia ou à l'enseignement à distance ne sont pas encore arrivées à un niveau de maturité satisfaisant. L'adaptation au e-learning est encore difficile, de multiples systèmes et langages existent, pour la plupart mal maîtrisés par les auteurs de contenu, enfin les réseaux multimédia souffrent entre-autres de problèmes de bande passante.

\section{LES DIDACTICIEL MICROWIND ET DSCH}

En partenariat avec ST-Microelectronics [1], nous avons développé un logiciel d'introduction à la conception micro-électronique CMOS dans un but résolument éducatif, sur PC [2]. Le but de l'outil est d'aider à la compréhension des phénomènes physiques à l'échelle des masques (Microwind) et des cellules logiques (Dsch). Le schéma de la figure 
1 positionne ces outils dans le contexte général de la conception de circuits intégrés.

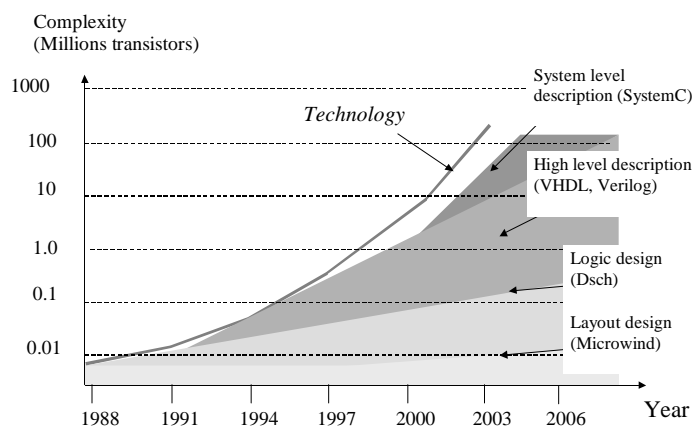

fig 2 : Evolution de la complexité et positionnement des outils Microwind et Dsch

Le rôle des didacticiels Microwind et Dsch est d'illustrer les phénomènes électriques à l'échelle des briques de base par le biais d'outils de conception et de simulation interactifs. L'étudiant dispose de vues attractives de la technologie, de simulations de la fabrication, de simulations statiques et dynamique $\mathrm{du}$ fonctionnement du circuit. Il fait ainsi le lien entre le dessin physique et les performances en temps, en consommation et en surface silicium. L'outil permet de visualiser l'impact d'un changement de technologique, notamment l'augmentation des vitesse de commutation, et les phénomènes parasites de fuite.
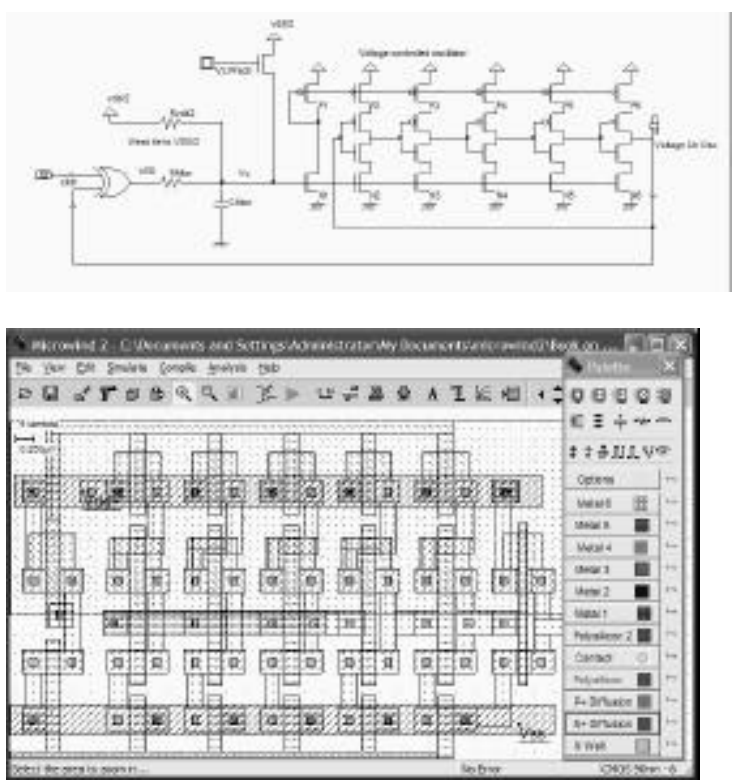

fig 3 : Schéma sous DSCH d'une PLL et écran principal de Microwind configuré en CMOS 90nm, pour la simulation analogique de la PLL
L'écran de la figure 3 représente le dessin des masques d'un oscillateur contrôlé en tension (VCO) destiné à une boucle à verrouillage de phase intégrée, en utilisant Microwind. Le schéma électrique créé avec DSCH est donné à titre informatif. Seules les parties logiques peuvent être simulées sous DSCH.

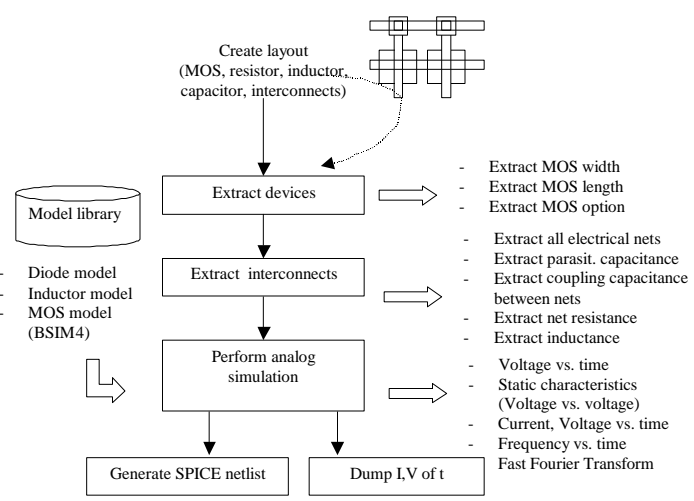

fig 4: Principe de l'extraction et de la simulation au niveau physique

Le logiciel Microwind intègre un outil d'extraction des paramètres électriques depuis le dessin des masques. Le détail des étapes d'extraction puis de simulation analogique est donné figure 4 . La simulation analogique repose sur une bibliothèque de modèles. Pour les transistors MOS, le modèle BSIM4 est disponible pour chaque technologique sub-micronique et nanométrique.

La simulation de type tension en fonction du temps est accessible sans recours à un simulateur externe. Un exemple de courbe obtenue dans le cas d'un travaux pratique de conception de circuits CMOS orientés radio-fréquences est donné figure $5 . \mathrm{La}$ boucle à verrouillage de phase dont le schéma correspond à la figure 3 est simulée au niveau transistors. Une fois la séquence de précharge réalisée, l'oscillateur se cale sur la fréquence de consigne fixée à l'entrée $(2.45 \mathrm{GHz})$.

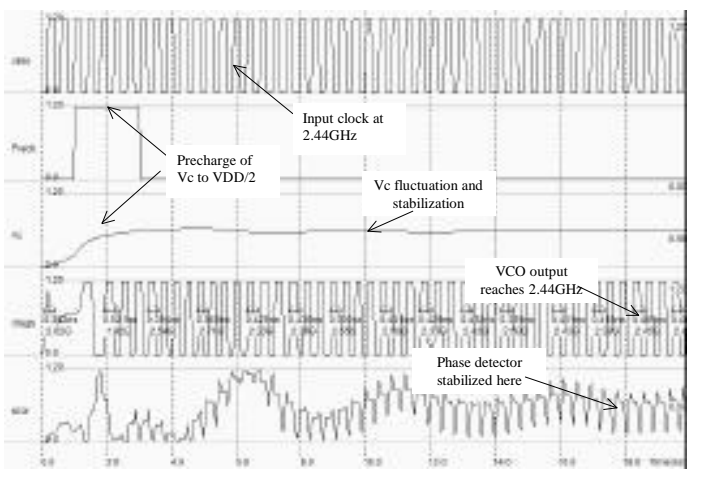

fig 5 : Simulation analogique de la PLL configurée pour $2.45 \mathrm{GHz}$ 
Les représentations du procédé en $2 \mathrm{D}$ et $3 \mathrm{D}$ ont un impact éducatif appréciable par l'aide visuelle qu'elles apportent et les questions qu'elle suscite. Le dessin du circuit fabriqué peut être modifié à loisir par l'utilisateur, une latitude très appréciée pour comprendre l'agencement du circuit et l'impact du dessin sur les performances du circuit (Figure 6).

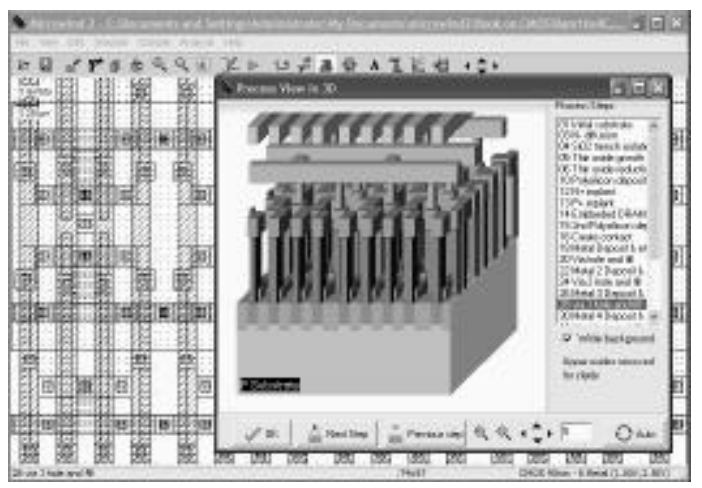

fig 6 : Vues $2 D$ et $3 D$ d'une mémoire SRAM en 90nm

\section{UTILISATION DE MICROWIND POUR L'ÉDUCATION}

Le logiciel Microwind est utilisé depuis de nombreuses années pour l'illustration des concepts fondamentaux de conception de circuits CMOS, dans de nombreuses universités et centres de formation. Il représente une introduction attractive et pédagogique, avant de passer à l'utilisation de logiciels industriels capables de concevoir des circuits de très grande complexité. Cependant, Microwind ne traite que le niveau physique de la micro-électronique, ne traitant donc pas des aspects de conception haut niveau.

Dans le cadre de l'illustration des cours de conception de circuits CMOS, Microwind est utilisé principalement en bureau d'études pour:

- La compréhension des transistors MOS: commutateur, caractéristiques I/V, comportement sous le seuil, options de MOS, différences entres modèles (LEVEL3, BSIM4)

- La conception et simulation de cellules de base. En CMOS 90nm, l'accent est mis sur le dimensionnement des transistors, les performances, les courants de fuites, les options de MOS (high speed, low leakage, high voltage)

- La propagation du signal: effet RC des interconnexions, routage des alimentations, rôle des niveaux métalliques

- La conception de mémoires: RAM, ROM, DRAM, SRAM, FRAM, EEPROM, FLASH. Microwind dispose des modèles de transistor double grille, et simule le comportement magnétique des matériaux utilisés dans les FRAM

- Conception d'amplificateurs, les oscillateurs, VCO, mixers, et convertisseurs. Par exemple
(Figure 7), un mixeur à base de cellule de Gilbert peut être simulé, avec une vérification de l'addition des fréquences d'entrée $(450 \mathrm{MHz}, 2 \mathrm{GHz})$ sur la sortie $(2.45 \mathrm{GHz})$ grâce à la transformée de Fourier (Figure 8).

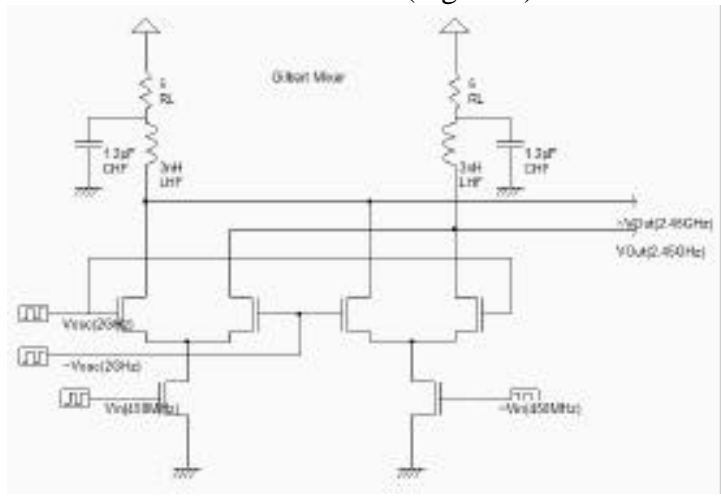

fig 7: Mixeur utilisé pour l'addition de fréquence fin $=450 \mathrm{MHz}$, fosc $=2 \mathrm{GHz}$

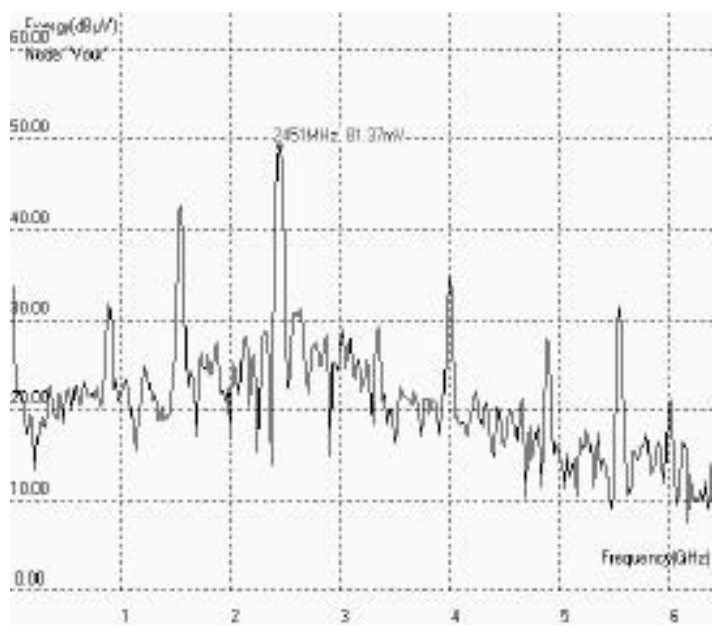

fig 8: Transformée de Fourier montrant la composante fosc + fin à $2.45 \mathrm{GHz}$

\section{CONCLUSION}

Les outils Microwind et Dsch ont été décrits dans cet article, avec une illustration en CMOS 90nm. Les logiciels évoluent chaque année au gré des avancées technologiques. Diffusés gratuitement sur le Web [3], Microwind et Dsch sont utilisés sur environ 500 sites. Deux livres sont en préparation, qui utilisent le logiciel comme base de travaux pratiques.

\section{Bibliographie}

1. International Rodmap for Semiconductors

2. http://www.mesdie.org

3. http://intrage.insa-tlse.fr/ etienne 